\title{
DEPICTIONS Y MINIFICCIÓN: UNA REFLEXIÓN SOBRE LA TRADUCCIÓN DEL MICRORELATO COMO DIDÁCTICA PARA LA FORMACIÓN DE INTÉRPRETES DE LENGUA DE SEÑAS EN COLOMBIA
}

\author{
Alex Giovanny Barreto* \\ UNAD - Colombia \\ Román Santiago Artunduaga** \\ UNAD - Colombia
}

\begin{abstract}
Resumen: El artículo presenta algunas reflexiones sobre una propuesta didáctica para la formación de intérpretes basada en la traducción enfocada en la competencia comunicativa. Esta experiencia inició en distintos talleres de la Asociación de Traductores e Intérpretes de Lengua de Señas de Colombia (ANISCOL) y ahora se han formalizado en el marco del proyecto curricular Licenciatura en Lenguas de Señas del grupo de Investigación UMBRAL de la Universidad Nacional Abierta y
\end{abstract}

* Estudiante de Maestría en Antropología, Universidad Nacional de Colombia (UN), Licenciado en Etnoeducación, especialista en Educación, Cultura y Política, Docente Ocasional Escuela de Ciencias de la Educación y Líder del proyecto curricular Licenciatura en Lenguas de Señas, con énfasis en lsc, de la Universidad Nacional Abierta y a Distancia - UNAD. Bogotá, Colombia. E-mail : agbarretom@gmail.com y alex.barreto@unad.edu.co

${ }^{* *}$ Doctorando en Filosofía, Universidad de Salamanca (España). Magister en Filosofía Latinoamericana, Licenciado en Filosofía y Lengua Castellana, Universidad de Santo Tomás. Líder de Investigación Escuela de Ciencias de la Educación de la Universidad Nacional Abierta y a Distancia - UNAD. Bogotá, Colombia. E-mail: roman.artunduaga@unad.edu.co 
a Distancia- UNAD. La propuesta didáctica está enfocada en el modelo de los esfuerzos (Gile), específicamente en el esfuerzo de producción y comprensión. Se presenta una crítica sobre la competencia traductora. La minificción es un género literario con múltiples posibilidades semióticas y filosóficas. Estos textos presentan un gran potencial para transmitir representaciones visuales, gestuales y espaciales de la lengua de señas colombiana, lo cual es útil para la formación de intérpretes. A través de la traducción de El Dinosaurio, concluimos con una mirada sobre de las potencialidades pedagógicas y didácticas de la minificción y los depictions en el diseño de actividades en la formación de intérpretes de lengua de señas.

Palabras clave: Depiction. Minificción. Interpretación de lengua de señas. Sordos. LSC.

\title{
DEPICTIONS AND MINIFICTION: A REFLECTION ON TRANSLATION OF MICRO-STORY AS DIDACTICS OF SIGN LANGUAGE INTERPRETERS TRAINING IN COLOMBIA
}

\begin{abstract}
The article presents reflections on methodological translationpractice approach to sign language interpreter's education focus in communicative competence. Implementing translation-practice approach experience started in several workshops of the Association of Translators and Interpreters of Sign Language of Colombia (ANISCOL) and have now formalized in the bachelor in education degree project in signed languages, develop within Research Group UMBRAL from National Open University and Distance of Colombia-UNAD. The didactic proposal focus on the model of the efforts (Gile), specifically in the production and listen efforts. A criticism about translating competence is presented. Minifiction is literary genre with multiple semiotic and philosophical translation possibilities. These literary texts have elements with great potential to render on visual, gestural and spatial depictions of Colombian sign language which is profitable to interpreter training and education. Through El Dinosaurio sign language translation, we concludes with an outline and reflections on the pedagogical and didactic potential of minifiction and depictions in the design of training activities in sign language interpreters.
\end{abstract}

Key words: Depiction. Minifiction. Sign language interpreting. Deaf. LSC 


\section{Competencias Traductoras e Interpretativas ${ }^{1}$ vs Competen- cias Comunicativas.}

Toda persona que es intérprete debe tener un dominio obligatorio de la gramática de las lenguas de trabajo. Es un consenso axiomático sobre la formación de intérpretes, dejando de lado las perspectivas semióticas o multimodales de la traducción que no implicarían estrictamente lenguas habladas o señadas. (cfr. Eco; e.g. traducir una obra musical en una pintura, o un cuento en una película, o las prácticas culturales en una etnografía). En Estudios de Traducción, la discusión sobre las competencias traslatorias como un dominio que está más allá de la comunicación ha sido propuesta inicialmente por la teoría funcionalista ${ }^{2}$ (action-theoretic approach, en inglés) de Reiss y Vermeer, y algunas de sus colegas (Witte; Holz-Mänttäri). En sus términos,

El iniciador no es un experto en traducción. Por ello, si se le pide que describa cómo debe ser el texto que necesita, se le está exigiendo algo que sobrepasa sus capacidades. (Holz-Mänttäri 368; apud Witte 47)

"[L]a [Teoría Funcionalista General de la Traducción] otorga al traductor la autoridad y el poder de decisión sobre la acción traslativa, al tiempo que le exige unas competencias específicas que le capacitan para tomar estas decisiones con la responsabilidad experta pertinente" (Witte 46) (el énfasis es mio)

Aunque las mismas autoras se han encargado de expresar sus postulados accionistas en términos de una versión de la teoría de la traducción como comunicación intercultural, en la práctica, su propuesta reduce la atención a la lengua sólo cómo una práctica cultural verbalizada (Witte 23,24 ) y se concentra en un supuesto saber traductológico específico que parece no hacer énfasis sobre uso y competencia de la comunicación. 
Esto ha llevado a una interpretación particular. La traducción no podría ser considerada como parte de la comunicación per se (Gutt 23). Gutt reacciona hacia la objeción del funcionalismo sobre los modelos de comunicación, y a su fuerte sentir sobre la traducción como un campo que cubre aspectos por encima de lo que cualquier otra disciplina puede hacer. Sin embargo, siguiendo a Gutt, es importante tener en cuenta lo siguiente: primero, Reiss y Vermeer se contradicen al promover su posición como multidisciplinaria y reducir al mismo tiempo escoger la conducta como el dominio de la teoría; si podemos circunscribir una 'competencia traslatoria' sin la necesidad de describir y clasificar el amplio rango de factores con respecto a los distintos dominios que conlleva esta competencia, no es necesario un acercamiento multidisciplinario a priori. Si el enfoque de las 'competencias traslatorias' son formuladas en una perspectiva realmente multidisciplinaria, deberían ser vistas en detalle, para empezar y sin apresurarse, desde varios enfoques más elementales, por ejemplo, un enfoque de competencias comunicativas (cfr. Hymes). Segundo, el funcionalismo ha mostrado las debilidades del modelo de comunicación para explicar la traducción. Esto es perfectamente válido. Sin embargo, el modelo críticado por esta teoría es el que Speber \& Wilson llaman el "modelo del código", una versión criticada por la Teoría de la Relevancia (ibíd.) en oposición al "modelo ostensivo inferencial". Así pues la diferencia entre Gutt y la propuesta de Reiss y Vermeer está derivada en gran manera por la concepción del modelo de comunicación asumida. Esta tensión se puede atenuar desde distintas perspectivas aparte de la tradicional semiótica, como la antropología lingüística (Duranti) o las ciencias cognitivas (Gutt). En palabras de Gutt,

\footnotetext{
"The fact that a particular approach to communication is inadequate does not necessaryly mean that any communicative approach [of translation] is inadequate" (Gutt 23)*.
} 
El llamado de atención de Gutt, es relevante en la medida en que no podemos sugerir arrojarle a las competencias traslatorias una autonomía completa de las competencias comunicativas.

Aparte de las supuestas competencias traductoras, el intérprete necesita ante todo debe ser un 'buen' comunicador en sus lenguas, tener un 'buen' dominio como hablante bilingüe. Ser intérprete es sinónimo de poseer ese dominio. Ya sea por haberlo desarrollado desde tierna edad como en el caso de la India Catalina que sirvió de intérprete para el conquistador Pedro de Heredia en la Ciudad de Cartagena (cfr. el concepto de Bi-texto y traducción natural de Harris retomado por Toury, 141), o ya sea por haberlo desarrollado posteriormente, ser intérprete implica ser reconocido por las mismas lenguas con las que se va a trabajar. Implica ser una India Lengua (Urbina Joiro) o sencillamente, un lenguaraz (Bértola y Fernández).

Sin embargo, la formación en lengua de señas para intérpretes tiene dimensiones específicas que las diferencian del ejercicio en otras lenguas habladas. Es muy usual que los intérpretes de lengua de señas acostumbren a trabajar, al contrario de sus colegas en lenguas orales, de su primera a su segunda lengua (Hauser, Finch y Hauser; Quinto-Pozo). Quinto-Pozo (Jacobs 213 apud QuintoPozo 177) ha señalado que existen observaciones anecdóticas que sugieren que un intérprete oyente nativo en inglés necesita comprometerse al menos 1320 horas para lograr un dominio de la lengua de señas americana (ASL) para "satisfacer la mayoría de trabajos que requieren el uso frecuente de la lengua, pero no siempre de forma aceptable y efectiva" ${ }^{3}$ (op. cit., 177), de modo que lograr la maestría en la lengua podría tomarle de 6 hasta 15 años. Así pues, la discusión sobre los medios efectivos para enseñar y verificar el aprendizaje de todos los niveles de proficiencia de una lengua, debe ser un tema central en los Estudios de Traducción e Interpretación de lengua de señas. 


\section{Traducción y filosofía: hipótesis de sentido.}

Abordaremos la traducción desde un paso filosófico previo: la comprensión. En los ámbitos y funciones del discurso, la comprensión forma parte tanto de la comunicación como de la estética de la traducción, bajo el supuesto de que la lengua de partida comparte con la lengua meta una estructura común básica. En efecto, en esta relación, es evidente al preguntar qué es lo que se traduce en una traducción; no son las palabras, las frases, las acciones, los escenarios, etc., sino la comprensión del texto/discurso, que es a un tiempo-unidad de comunicación y unidad de traducción (UT) (Nord).

La traducción es un hecho hermenéutico en la medida en que sobre ella gravita la aprehensión del sentido. Debido a esto, el proceso de la comprensión, como el de la traducción, ha sido signado por la tradición de un carácter subjetivo, en especial por la filosofía hermenéutica del romanticismo. En efecto, para Schelling, comprender es sacar a luz la fuerza inmanente, el espíritu, -como sostuvo Hegel-, que puja por su descubrimiento en la esfera íntima de la conciencia para manifestarse luego en el concepto y en el sistema. Asimismo, para Dilthey, la comprensión se relaciona con el sentido que el intérprete da a los elementos que componen la "trama de su vida". En contraste, el positivismo lógico de principios del siglo XX pretendió encontrar un sustrato objetivo en la estructura lógica del lenguaje. Designar algo con el lenguaje es ya reconocer la existencia de algo (un "X") fuera del sujeto (objectum). Sin embargo, dicha empresa conllevaba paradojas y contradicciones. Con el segundo Wittgenstein, Austin, Searle y el descubrimiento de la influencia (¿determinación?) del contexto y de la centralidad de la cultura en el lenguaje, la pregunta por el sentido, si bien no regresa a sus cauces de subjetividad, reconoce marcos de conocimiento a los que el intérprete y traductor recurre para comprender y para transmitir el contenido de su comprensión. Con todo, no se tratará de fuerzas ocultas que se manifiestan en la realización del discurso, ni objetividades arbitrarias de la mente, sino del resultado de la 
interacción comunicativa. Con lo anterior, queremos dejar sentado que una traducción está referida a niveles de comprensión cada vez mayores, no sólo de lo que quiso decir el autor/emisor, sino de la "intención" que buscaba con su discurso en un contexto determinado, es decir, se trata de discernir la función del discurso en un "ámbito de la realidad" (económico, político, religioso, técnico, educativo, gubernamental etc.) y su función en un contexto específico. Identificar el ámbito del discurso y sus funciones constituye un primer momento del ejercicio de la traducción.

La comprensión es un proceso cíclico en donde interviene información. Esta información se manifiesta en oraciones. Dichas oraciones, en su secuencialidad, generan expectativas de sentido, donde el ámbito del discurso tiene su función particular. Las oraciones, individualmente tomadas, son el contenido de esa expectativa de sentido, pero dicha expectativa es una reconstrucción, es decir, se forma a medida que una oración sigue a la otra. Es entonces cuando se obra una abducción semántica, donde el todo es construido por las oraciones particulares y estas adquieren horizonte de sentido en virtud de la totalidad. La expectativa de sentido se ve con claridad, por ejemplo, en los chistes, donde el efecto cómico es precisamente la suspensión de la expectativa construida por las oraciones precedentes a la conclusión.

Las funciones del discurso descansan en la intencionalidad que se quiere con el discurso. Para identificar esa intencionalidad juega un papel destacado el contexto comunicativo y el marco de conocimiento. La intencionalidad viene a complementar la expectativa de sentido referida a un objetivo esperado. Por ejemplo, pensemos en la situación específica en una clase de posgrado en literatura, de la que hablaremos más adelante. En esta situación nos encontramos con un ámbito del discurso que responde a reglas culturales y sociales específicas. La intencionalidad, en este caso, es la de enseñar un contenido, así que los destinatarios (los estudiantes de posgrado) dan sentido a las oraciones dichas o escritas cuando se reconoce esa intencionalidad. La expectativa de sentido es calibrada por la coherencia que tiene la sucesión de las oraciones y esta a su vez está marcada 
por la intencionalidad. La función del discurso tiene que ver con los objetivos intencionales que persigue (pragmática), el marco de conocimiento y el ámbito específico del discurso (semántica).

La serie de oraciones de un discurso debe ser sometida organizada y reducida por el traductor, esto es, convertirla en secuencia. Para esto el traductor precisa de su memoria semántica funcional o a corto plazo (MCP). Con el trabajo de traducciones posteriores el traductor va asociando estas experiencias a nuevos eventos comunicativos mejorando en las competencias de comprensión. Ante un nuevo ejercicio de traducción el intérprete-traductor llena los espacios omitidos gracias al marco de conocimiento apoyado de su memoria a largo plazo (MLP).

Como señala Derrida, todo escribir supone algo que se ha borrado, es decir, que en la comprensión de lo escrito está presente lo omitido, lo editado, lo corregido, lo ausente. En este orden de ideas, la teoría de la narración señala la importancia de un análisis "formal", estructural, de la narración para identificar la intención del autor manifestada en la escogencia de los hechos narrados en donde se pueda identificar una intencionalidad (la presencia de una ausencia). La narración dentro de una trama es una clave de comprensión del enunciado del autor. En la medida en que el autor aporta sentido al reiterar un hecho, pasar rápidamente a otro, elegir un determinado registro, iniciar o concluir con tal o cual hecho, etc., la narración participa de los actos ilocutivos. Estimar las particularidades del discurso narrativo constituye en propiedad una competencia comunicativa.

La traducción de un micro-relato adquiere un papel didáctico importante para el traductor, pues le permite lanzar hipótesis de sentido o identificar más eficientemente los hilos de la narración. Igualmente, la brevedad de las narraciones de este tipo posibilitan evaluar el nivel perlocutivo de la narración, pues el traductor está al mismo nivel que el receptor, participando de 'efecto' de la narración de la misma manera que, dentro del 'marco común de conocimiento', lo hace el destinatario. Esto supone a su vez un conocimiento por parte del traductor de la estética de la na- 
rración, elemento mediante el cual se enriquece la comprensión del discurso.

\section{La Minificción: Vívidas hipótesis de sentido}

La minificción es un género reciente en la historia literaria. Aunque tiene expresiones en distintas lenguas, su formulación se consolidó en el siglo XX en Hispanoamérica, donde en particular los autores de México se han arrojado su nacimiento (Zabala). El género se caracteriza porque produce textos que rara vez rebasan una página impresa (cuento corto 2000 a 1000 palabras), un párrafo (cuento muy corto, de 100 a 200 palabras) o incluso, una oración (cuento ultracorto, de 200 a 1 palabra). El naciente género está ampliamente relacionado con otros géneros clásicos breves como el aforismo, el haiku, epigrama, soneto entre otros, pero se caracteriza porque son relatos que al usar múltiples recursos literarios específicos (la elipsis, la condensación, la alusión, la anáfora etc.) logran un efecto de complejidad en medio de la sencillez textual. En general se escriben un conjunto de ellos, los cuales mantienen una línea argumental supertextual.

En general, se asume que la minificción enfrenta retos y problemas teóricos a nivel literario y lingüístico, por lo menos en relación a seis áreas propias de los tiempos contemporáneos: brevedad, diversidad, complicidad, fractalidad, fugacidad y virtualidad (Zabala 58 - 71). Un análisis de estos problemas ha revelado la profunda relación de este tipo de escritura en relación con la posmodernidad; el género es un reflejo de la pérdida de esencias discernibles, el cuestionamiento de la autoridad, la reconstrucción social de la realidad, el descrédito creciente del orden racional, una reflexión individual irónica (Jarrín Ballesteros).

Por supuesto, para comprender la compleja dimensión que proponen los microrelatos hace falta leerlos y traducirlos. Un buen ejemplo es el que propone Luis Felipe Hernández en su Circo de tres pistas y otros mundos mínimos 


\section{Escena Conyugal}

Lanzaba con presteza uno tras otro los cuchillos a su mujer quien los recibía con el trapo para secarlos (53)

Una escena cotidiana fugazmente se convierte y re-convierte de una escena circense frente a los ojos del lector. Desde el título, el lector, condiciona ciertas "hipótesis de sentido", sobre lo que es una escena conyugal, pero rápidamente el texto lo lleva a elemento macro textual del título donde está inmerso el relato (el circo) para luego, con ironía, devolverlo a la cotidianidad. Es un juego fugaz.

También estos relatos juegan con los posibles "paseos intertextuales" (Eco) que pueda ser un lector en su conocimiento enciclopédico. Sólo hace falta un relato como el de José Emilio Pacheco,

\section{La Lechera}

La lechera hacia proyectos mientras caminaba por la ciudad. De pronto, ella, su jarra y sus ilusiones se volvieron añicos en la explosión nuclear (73)

En este caso, rompiendo espacios, temporalidades y tradiciones, de manera repentina, Pacheco logra darle un giro sutil y crítico a un relato conocido.

Por supuesto, este tipo de recursos son posibles en la medida que su usen recursos de la lengua española. ¿Qué tipos de recursos serían potencialmente útiles para expresar y traducir la minificción a una lengua de señas?

\section{Señas Policomponenciales y Depictions en LSC.}

Las señas policomponenciales, o "clasificadoras" (Slobin, Hoiting y Kuntze; Schembri; Quinto-Pozos; Liddell) son unidades en la lengua de señas que representan acciones, agentes e imagineria en una fusión entre elementos linguisticos de la lengua de señas 
(Ilustración 1) y elementos gestuales y espaciales (Ilustración 2). Estos elementos son uno de los aspectos en los que se ha documentado más dificultad en el aprendizaje de la lengua de señas como segunda lengua para adultos (Quinto-Pozo 177) y una adquisición que los niños sólo llegan a dominar a una edad posterior a los 8 años (Galvan, Schick, apud. Emmorey, 194).

La ilustración 1 y 2 fueron extraidas de dos narrativas elicitadas en lengua de señas colombiana por un sordo hijo de sordos, de mediana edad y una región caribeña del país, a partir de la observación del video mudo Pears Story ${ }^{4}$. En la primera parte de la ilustración 1, el brazo y la mano izquierda codifica un árbol, mientras que la mano derecha codifica una persona de pie. En la frase-seña, la mano derecha se acerca y hace contacto con el antebrazo, codificando la acción "una persona se acerca al árbol”. En la otra parte, las formas de las manos y acción codifican "alguien, se acerca a dos personas". Se ha observado que los "clasificadores" manuales de persona, tienen distintas formas entre lengua de señas (e.g. el pulgar y el meñique en lengua de señas japonesa (JSL), para codificar un hombre o una mujer o el indice curvo en ASL para representar una persona caminando). Así pues, este tipo de señas son tan complejas que resulta díficil clasificarlas como verbos, clasificadores o incluso, predicados (Schembri).


Ilustración 1: Los predicados policomponenciales "alguien-se-acerca-a-un-árbol" y "alguien se acerca a dos personas" 



Ilustración 2: Predicado policomponencial "subirse-a- la bicicleta"

Algunas de estas señas, como en la Ilustración 2, incluyen gestos. En la primera y segunda secuencia se observa el gesto de alguien tomando una bicicleta por los manurios ${ }^{5}$. En la tercera secuencia, se incorpora una forma de la mano en forma de persona que se sienta en la bicicleta. Estos ejemplos, revelan que los predicados realizados con estas señas tienen una construcción que al mismo tiempo puede ser lingüística y gestual (Oviedo).

Ahora bien, la formación en lengua de señas de los intérpretes se enfoca en un nivel textual y discursivo. A nivel del texto y el discurso, los predicados policomponenciales tienen formas complejas en las que pueden transmitir significado, mezclandose con elementos gestuales, espacial y por lo tanto, con imagineria (Imagery) visual (crf. con el concepto vigoskyano de Growth Point aplicado a las lenguas de señas; McNeil). Paul Dudis, bajo los principios de la lingüística cognitiva, ha avanzado en una clasificación sobre las posibilidades esquematicas entre señas y predicados componenciales, espacio, gestos e imagineria. El autor los denomina depictions y los nombra con códigos específicos (ver tabla 1) y un esquema (Ilustración 3) de sus componentes de representación en términos de gramática cognitiva (Langacker). 


\begin{tabular}{|c|c|c|c|c|c|c|}
\hline \multirow[t]{2}{*}{$\begin{array}{l}\text { Una } \\
\text { experiencia } \\
\text { del |self| } \\
\text { (Mostrada a } \\
\text { Tamaño } \\
\text { Real) }\end{array}$} & $\begin{array}{l}\text { ¿Lo que } \\
\text { dice un } \\
\mid \text { self } \mid \text { ? }\end{array}$ & $\begin{array}{l}\text { ¿Lo está } \\
\text { haciendo un } \\
\text { |self|, o una } \\
\text { |parte| de él? }\end{array}$ & $\begin{array}{l}\text { ¿Hay |otras } \mid \\
\text { representaciones } \\
\text { visuales, además } \\
\text { del } \mid \text { self|? }\end{array}$ & $\begin{array}{l}\text { ¿Un |Self| } \\
\text { está siendo } \\
\text { representado } \\
\text { de forma } \\
\text { simultánea } \\
\text { por la } \\
\text { |mano|? }\end{array}$ & $\begin{array}{l}\text { ¿Es lo que un } \\
\text { |self| está } \\
\text { pensando, } \\
\text { pero que no } \\
\text { es visible en } \\
\text { potencia por } \\
\text { |otro|? }\end{array}$ & $\begin{array}{l}\text { ¿Es un } \\
\text { evento } \\
\text { comprimido } \\
\text { en el tiempo } \\
\text { percibido } \\
\text { por un |self?? }\end{array}$ \\
\hline & Código A & Código B & Código C & Código D & Código E & Código $F$ \\
\hline \multirow[t]{2}{*}{$\begin{array}{l}\text { Una } \\
\text { experiencia } \\
\text { sin |alguien| }\end{array}$} & $\begin{array}{l}\text { ¿Es una } \\
\text { |evento } \mid \text { a } \\
\text { tamaño } \\
\text { natural? }\end{array}$ & $\begin{array}{l}\text { ¿Es un } \\
\text { |evento| } \\
\text { comprimido } \\
?\end{array}$ & $\begin{array}{l}\text { ¿Es una |escena| } \\
\text { u |objeto a a } \\
\text { tamaño natural? }\end{array}$ & $\begin{array}{l}\text { ¿Es una } \\
\text { |escena| u } \\
\text { |objeto| } \\
\text { concreto } \\
\text { comprimido } \\
?\end{array}$ & & \\
\hline & Código G & Código $\mathrm{H}$ & Código I & Código J & & \\
\hline \multirow[t]{2}{*}{$\begin{array}{l}\text { Una } \\
\text { |abstracción } \\
\text { | }\end{array}$} & $\begin{array}{l}\text { ¿Es un } \\
\text { plano } \\
\text { calendario } \\
?\end{array}$ & $\begin{array}{l}\text { ¿Es una lista } \\
\text { de boyas? }\end{array}$ & $\begin{array}{l}\text { ¿Es una línea de } \\
\text { tiempo? }\end{array}$ & $\begin{array}{l}\text { ¿Es } \\
\text { token? }\end{array}$ & & \\
\hline & Código K & Código L & Código $\mathrm{M}$ & Código N & & \\
\hline
\end{tabular}

Tabla 1: Resumen de los códigos tomada de los depictions tomada de Cortés basada en Dudis. Los nombres entre barras son usados siguiendo la propuesta de Liddell para representar espacios mentales fusionados en lengua de señas (Liddell). Típicamente lo que se conoce como Diálogo-Acción Construida (Metzger) en este esquema sería respectivamente el código Ay B.

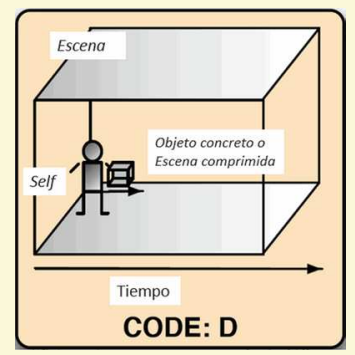

Ilustración 3: Descripción de componentes de esquema de un depiction "Código D” inspirado de Dudis (Depiction Identification Flowchart version 4.9.1).

En este artículo asumiremos los depictions como un elemento de cohesión discursiva que no reduce a elementos léxicos. Incluye 
la imaginería, las gestualidad y el espacio, en una visión más amplia que otros abordajes como el dialogo-acción construida (Metzger) o el role-shifting (Padden). Bajo la propuesta de Dudis, es posible organizar los depictions en relación con otros fenómenos, tal como se ha hecho, por ejemplo, con la adquisición de lengua de señas (Langdon).

\section{Traduciendo la minificción: Una microrelato a modo de ejemplo.}

A través del proyecto Licenciatura en Lengua de señas, hemos pensado en las múltiples opciones que tiene la minificción como un elemento temático para la construcción de estrategias para la enseñanza de las señas policomponenciales y la cohesión textual a través de depictions en la LSC. En el marco de enfoque de diseño curricular en educación superior por competencias (Tobón), queremos ilustrar los componentes elementales de lo que podría llegar a ser una propuesta didáctica entre depictions y minificción a través de una actividad puntual: la traducción de una de las obras cumbre de la minificción en habla hispana, El Dinosaurio de Augusto Monterroso (75).

\section{El Dinosaurio}

Cuando despertó, el dinosaurio todavía estaba allí.

Hemos escogido El Dinosaurio convenientemente, por ser uno de los microrelatos conocidos más breves pero al mismo tiempo más complejos a nivel semiótico (Una deuda retomada del análisis de Eco). Sin lugar a dudas, la traducción a lengua de señas de $E l$ Dinosaurio debe responder a las características del mini-relato en español para que pueda ser considerado 'casi lo mismo'.

Para implementar una sola actividad de traducción de un minicuento, en aras de fortalecer la formación de intérprete de lengua de señas, es necesario por lo menos abordar previamente otros 
objetivos de aprendizaje cómo prerrequisitos en un programa más extenso (un taller, un curso o un semestre universitario) que no ha sido tratados en profundidad aquí:

i. Reconocer y aplicar la teoría de los espacios mentales (Fauconnier) al análisis de diversos relatos y textos.

ii. Reconocer los enunciados cómo un conjunto de supuestos mutuamente manifiestos en el esquema del modelo ostensivo-inferencial de la comunicación (Speber y Wilson).

iii. Identificar las características de la minificción (González Martinez; Zabala) como género hipertextual y contemporáneo.

iv. Analizar las potencialidades de las señas policomponenciales (Emmorey) para la producción de múltiples significados simultáneos.

v. Analizar las potencialidades del Dialogo-Acción Construida (Metzger) en la producción de múltiples significados simultáneos.

vi. Producir textos en lengua de señas que usen apropiadamente señas policomponenciales y el Dialogo-Acción Construida

vii. Identificar los componentes esenciales desde la gramática cognitiva (Langacker) para la descripción de los depictions (Dudis)

Partiendo de las diferencias de la interpretación de lengua de señas en oposición con la traducción (Stone) (Ilustración 4), se partirá desde un 'contexto de situación' específico (Malinowsky, apud. Duranti, 293; compárese con 'Entorno cognitivo' de Speber y Wilson) que implicaría una 'interpretación a vista' situada entre la traducción y la interpretación de lengua de señas (ver ilustración 5) 


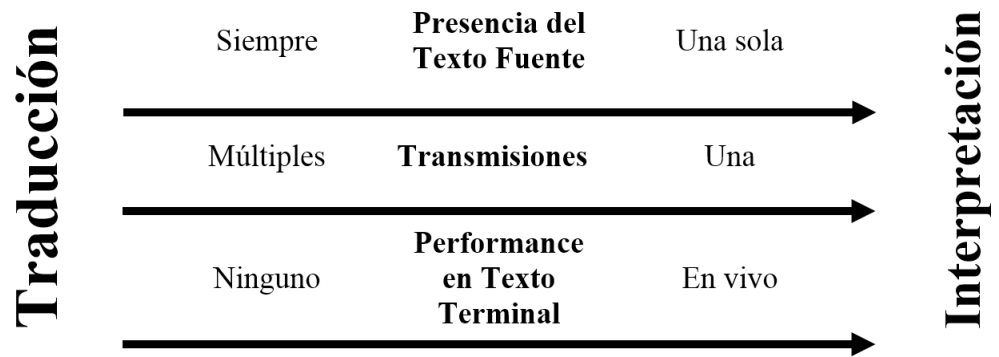

Ilustración 4: Continuum entre traducción e interpretación inspirado en Stone.

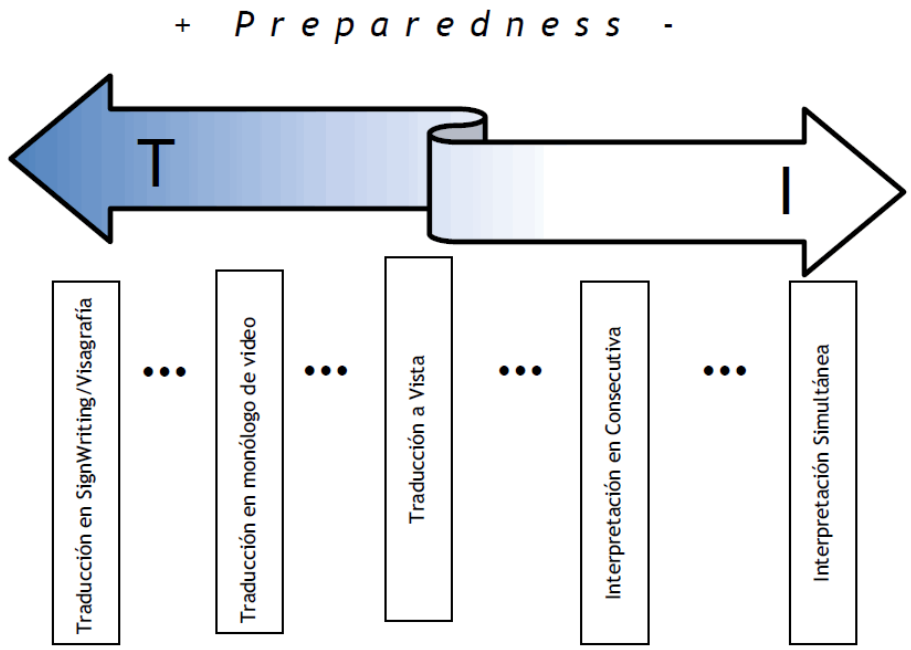

Ilustración 5: Modalidades de Traducción e Interpretación en continuum (Barreto y Bustos 219)

Situación Específica

El Dinosaurio se enuncia en un servicio de interpretación en un curso post-gradual, en donde el intérprete puede hacer la 'interpretación' del texto a la lengua de señas a un estu- 
diante avanzado de literatura. No se cuentan con fuentes bibliográficas al alcance y el intérprete sólo parte su experiencia de interacción con el estudiante sordo y de lo que dice el texto.

En estos términos, es indispensable que el intérprete identifique los supuestos contextuales más elementales o 'implicaturas' del micro-relato. Al respecto Umberto Eco señala dos:

i. Quando acordou, o dinossauro que tinha tentado ignorar dormindo-se, ainda estava lá ii. Quando acordou, o dinossauro com o que tem sonhado, ainda estava lá

(Eco, apud Barreto Muñoz)

La segunda implicatura parte de la base que el sueño es un espacio mental determinado, en este caso real, y no necesariamente un mundo posible abierto a todas las interpretaciones posibles de un 'paseo intertextual' (Eco, Seis Paseos Por Los Bosques Narrativos). De este modo, las implicaturas de El Dinosaurio se diferencian en su forma más básica por la forma en que se representa el dinosaurio, (i.e. el dinosaurio de un sueño, o un dinosaurio real). Esto se puede observar con facilidad en los esquemas de las relaciones de los espacios mentales de las implicaturas (Ilustraciones 6 y 7 ) 


a': 'Pessoa” no
passado.
$a_{1}$ : 'Pessoa'
dormindo
$a_{2}$ : 'Pessoa'
Real
d' dinossauro
no passado
d: dinossauro
R1: Espaço Real
(dormindo)
R2: Espaço Real
(acontecido)
M: Espaço do
passado



Ilustración 6: Esquema de espacios mentales de Implicatura 1 tomada de Barreto Muñoz.

a': 'Pessoa” no
passado.
$a_{1}$ : 'Pessoa'
dormindo
$a_{2}$ : 'Pessoa'
d' dinossauro
no sonho
d: dinossauro
R1: Espaço Real
(dormindo)
R2: Espaço Real
(acontecido)
M: Espaço do
passado

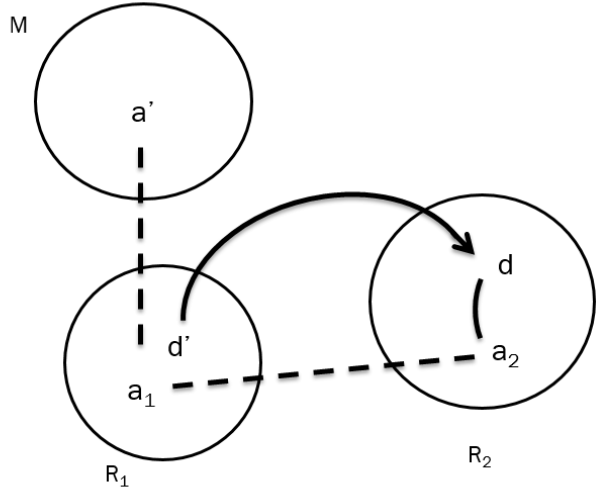

Ilustración 7: Esquema de espacios mentales de implicatura 2 tomada de Barreto Muñoz 
En los talleres realizados con esta propuesta didáctica, se ha observado de forma anecdótica que muchos intérpretes establecen espacios mentales elementales que no rescatan las dimensiones textuales de la minificción. Básicamente, esto sucede porque muchos tienen dificultades para establecer espacios mentales o mapeos complejos (Winston) en la comprensión de los textos y aún más, muchos presentan dificultades para representarlos en el discurso a través de distintos recursos en lengua de señas (e.g. señas policomponenciales, role-shifting, tópico-comentario) que nosotros agrupamos aquí en depictions.

\section{EI Dinosaurio en LSC: algunas observaciones}

Se han observado distintas estrategias para la traducción del Dinosaurio a la $\mathrm{LSC}^{6}$. La versión más común, es la que trata de seguir el orden lineal de los espacios mentales construidos. $\mathrm{M}=\{\mathrm{Se}$ sabe que hay un dinosaurio $\}, \mathrm{R}_{1}=$ Alguien que está en un sueño se despierta $\}$ y $R_{2}=\{$ Ese alguien se percata que el dinosaurio de $\mathrm{R}_{2}$ es el mismo de $\mathrm{R}_{1}$ \}. Tal como se observa en la ilustración 8 y 9 .

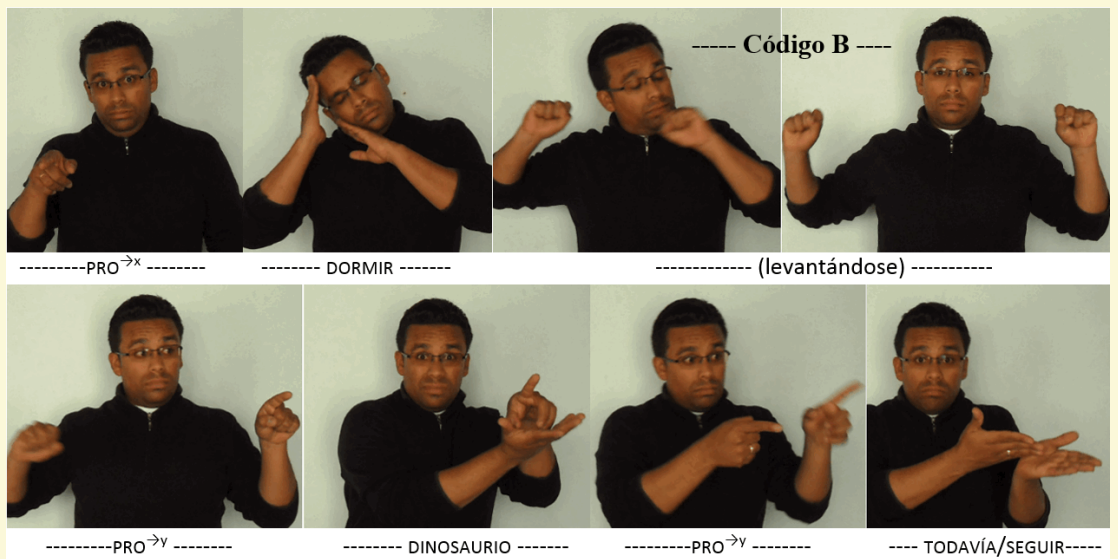

Ilustración 8: El Dinosaurio versión 1, agradecemos a Nicolás Sánchez por su contribución. 




Ilustración 9: El Dinosaurio versión 2, agradecemos a Yuly Gutierrez por su contribución.

Algunas de estas versiones pueden ser, en sí mismas complejas, debido a las posibilidades de los depictions. En la versión 2, la intérprete simula un dialogo interno de la persona que se despierta y por la posición en que está se puede asumir que la persona está en un lugar elevado ya que se describe el dinosaurio como 'grande' (el dinosaurio no es uno en miniatura).

Una vez que se socializa con los intérpretes las potencialidades de los depictions y la naturaleza de la minificción, se evidencia ejercicios mucho más complejos que tratan de imitar una presunta 'esfericidad' y 'brevedad' en lengua de señas, cómo lo muestra la ilustración 10, en donde se intenta en una sola escena ${ }^{7}$ incluir espacios mentales del Dinosaurio. 




(despertándose + dinosaurio $+\infty)$

Ilustración 10: El Dinosaurio versión 3, agradecemos a Nicolás Sánchez por su contribución

Los estudiantes de los talleres intentaron versiones más oníricas del microrelato. En la ilustración 11, una intérprete intentó a través de una 'partición del cuerpo' (Dudis, The body in scene: depictions, 26), simular la puesta de dos escenas: el self observando y el dinosaurio. Este tipo de apuesta es más compleja, en la medida en que los elementos del depiction pueden ser interpretados de múltiples formas que no se pueden determinar $(\infty)$. El dinosaurio en una esquina de la escena, en particular, puede interpretarse como un mini-dinosaurio que está cerca a un self que no se haya percatado de ello, o de un supuesto "recuadro" que represente un dinosaurio de tamaño real o no, de un espacio mental anterior. En este caso, esta versión podría referirse a la la segunda implicatura referida con anterioridad: 'Quando acordou, o dinossauro com o que tem sonhado, ainda estava lá'. El dinosaurio de la esquina, sería el dinosaurio del sueño. 


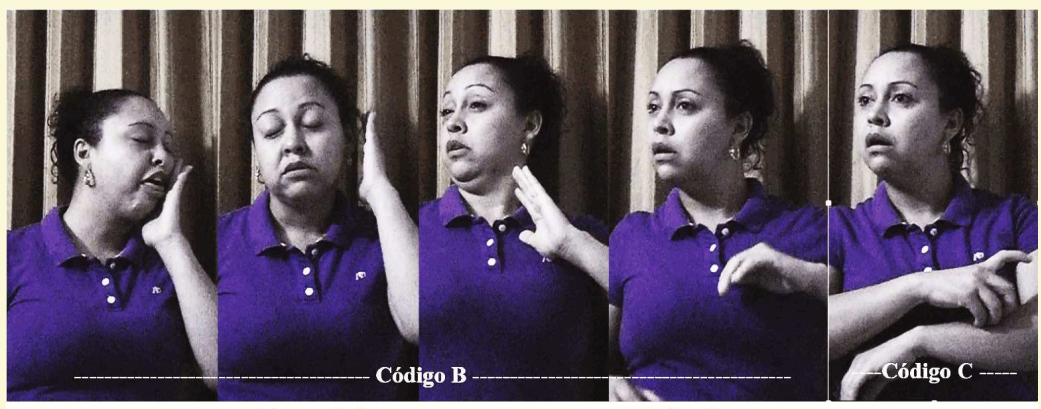

- (despertándose)

-- (mirando $+\infty)$---

mirando + dinosaurio $+\infty)$

Ilustración 11: El dinosaurio versión 4 agradecemos a Azucena Jiménez por la contribución.

\section{Proyecciones y Limitaciones}

El presente artículo está focalizado en cómo el ejercicio didáctico de traducción puede contribuir a la formación integral de intérpretes de lengua de señas. Por supuesto, la traducción de microrelatos podría contribuir a una reflexión más amplia sobre la formación de los traductores de lengua de señas, asunto que no se aborda en este escrito. Esta reflexión está estrechamente relacionada con la discusión sobre la relación de la competencia comunicativa bilingüe con la competencia traductora.

Un ejercicio de reflexión sobre las posibilidades de trasvase tanto en el esfuerzo de comprensión de un texto en español y producción de un texto en LSC, contribuye al fortalecimiento de la lengua. En términos del Gravitational Model of Language Availability (Ilustración 12, Gile, 226), el estímulo de textos en español con complejos espacios mentales y depictions en lengua de señas, activa un 'efecto centrípeto' que contribuye a que los intérpretes recuerden mejor constituyentes específicos de la lengua de señas (language constituents, en palabras de Gile, i.e. unidades léxicas, reglas composicionales de lenguaje no especializado y reglas de lenguajes para propósitos especiales). Como los depictions se 
presentan como un tipo amplio de cohesión discursiva sincrónicadinámica a nivel cognitivo, sus elementos (i.e señas o predicados policomponenciales, imaginería, iconicidad-metáfora y gestualidad), aunque no podamos decir que se corresponden con exactitud a los language constituents propuestos por Gile, podrían estar más disponibles como recursos para el ejercicio de los intérpretes, mejorando su desempeño.



Ilustración 12: Esquema inspirado en el Modelo Gravitacional de Disponibilidad Lingüística (Gile) aplicado a la formación de intérpretes de lengua de señas a través de depictions. Una propuesta de traducciones de mini-relatos establecería un estímulo que de forma 'centrípeta' contribuiría que recursos complejos de la LSC estuvieran disponibles a los intérpretes en su trabajo.

Sin embargo, este tipo de ejercicios pondría a prueba lo que una comunidad de sordos consideraría aceptable como una traducción literaria de un minirelato en lengua de señas. De hecho, implicaría “crear" un nuevo género o tipo discursivo en la LSC, en la medida que el concepto de minificción no está ampliamente socializado entre los sordos, ni siquiera entre los sordos de ciertos círculos 
profesionales. En la concepción de una Norma Sorda de Traducción (Toury, apud. Stone; Souza, Barreto A. G., Preservation of Sign Language (Veditz, 1913)), este tipo de tipología discursiva, estaría atravesado por múltiples factores que además de una estructura lingüística determinada, estaría relacionado con diversos tipos de relaciones y prácticas sociales de los sordos de Colombia. También, el uso de los depictions tendrían restricciones de tipo fono-morfológico que ya se han empezado a documentar en investigaciones recientes (Thumann, Identifying depiction: Constructed Action and Constructed Dialogue; Thumann, Identifying Recurring Depiction in ASL Presentations; Quinto-Pozos) lo que implicaría que un uso restringido en didácticas de formación de intérpretes y traductores de acuerdo a los textos específicos y a las posibilidades de traducción de las mini-relatos. Futuras investigaciones tendrían que verificar y confirmar estas limitaciones.

En el proyecto curricular Licenciatura en Lengua de Señas de la UNAD, pensamos que a pesar de las restricciones y limitaciones que se vayan comprendiendo con investigaciones lingüísticas, los Depictions tienen un potencial enorme en el planteamiento de competencias básicas en la formación de lengua de señas colombiana a como segunda lengua, en este caso, a personas que estén en proceso de formación también como intérpretes.

\section{Notas}

1. El funcionalismo acuño traslación como neologismo para abarcar la abstracción teórica común entre traducción e interpretación. Aunque consideramos útil este término, preferimos seguir distinguiendo entre traducción e interpretación, no obstante estemos de acuerdo que traducción e interpretación pertenecen a un solo continuo. 
* "El hecho que un abordaje particular de la comunicación no sea apropiado no significa necesariamente que todo abordaje comunicativo [de la traducción] sea adecuado"

2. Aunque se ha debatido sobre las diferencias hacen difícil hablar de una teoría "funcionalista" homogénea, incluso entre las mismas Reiss y Vermeer, en este artículo se llama funcionalista a la propuesta de estas autoras.

3. "satisfy most work requirements with language use that is often, but not always, acceptable and effective"

\section{4. http://pearstories.org/pears video.htm consultada el 11 de febrero de 2015}

5. La forma de las manos al tomar la bicicleta no es completamente icónica (no se podría tomar la bicicleta con los puños cerrados). De ahí que algunos autores podrían llamar a esta seña un clasificador de 'objeto manipulado' (Oviedo, 2001)

6. Agradecemos amablemente a la Asociación Nacional de Traductores e Intérpretes de Lengua de señas (ANISCOL) por la provisión de los ejemplos de traducciones usadas en este artículo.

7. El análisis de este tipo de depiction, es complejo en la medida que hay duda si la mano que representa el dinosaurio, tiene una línea de tiempo independiente a la representación del self. Este factor constituiría una diferencia entre los códigos C y D. En el caso de la ilustración 10, es más probable que el dinosaurio se inscriba en la misma línea del tiempo del self, mientras que en la ilustración 11, es difícil establecer eso. En todo caso, es interesante que las manos no representan una parte asociada al self, (e.g. cuando una persona camina o conduce y el rostro muestra su expresión facial, pero la mano representa sus piernas, una parte de su cuerpo o el vehículo en donde va o conduce), así que este elemento pudiera ser una confirmación de las categorías de Dudis (en el caso que no tengan una línea de tiempo aparte, si no están asociadas al self), o un ampliación de la categoría del Código D (i.e. que incluya Depictions que tengan una línea de tiempo independiente, pero que no estén asociadas con el self). 


\section{Referencias}

BARRETO MUÑOZ, A. G. (2013). Depictions, Espaços Mentais e Coesão na Tradução e Interpretação de Lingua de Sinais. Taller, APILDF-Universidade de Brasilia, Facultade de Educação, Brasilia.

BARRETO MUÑOZ, A. G. (en edición). Preservation of Sign Language (Veditz, 1913): Uma Leitura das Normas Surdas de Tradução para America Latina.

BARRETO MUÑOZ, A., \& BUSTOS, O. (2012). Teorias de la Traducción/ Interpretación en plastilina. Bogotá: Ediciones ANISCOL.

BÉRTOLA, C., \& FERNÁNDEZ, A. (2011). Notas para el estudio de las voces intérprete, ladino y lenguaraz en territorio oriental (siglos XVII a XIX). V Seminario sobre lexicología y lexicografía del español y del portugués americanos: a 200 años del inicio del proceso independentista del Uruguay. Montevideo: Academia Nacional de Letras.

CORTÉS, Y. (2013). Un acercamiento a los depictions como elementos constitutivos de los textos expositivos y argumentativos en LSC: implicaciones curriculares. Recuperado el 25 de junio de 2015, de Fenascol: http://www.fenascol.org. co/SEDasignaturaLSC/doctos/INVESTIGACION_LINGUISTICA.pdf

DERRIDA, J. (1998). De la gramatología. Madrid: Siglo XXI.

DILTHEY, W. (1890 [2010]). Wilhelm Dilthey: Selected Works: Understanding the Human World (Vol. II). (R. A. Makkreel, \& F. Rodi, Edits.) Pricenton University Press.

DUDIS, P. (2008). Depiction Identification Flowchart version 4.9.1. Recuperado el 3 de Abril de 2013, de Gallaudet Facultu-Staff Profile: http://www.gallaudet. edu/Documents/flowchart\%20version\%204.9.1.pdf

DUDIS, P. (2011). The body in scene: depictions. En C. Roy (Ed.), Discourse in Signed Languages. Washington: Gallaudet University Press. 
DUDIS, P. (2007). Types of Depiction in ASL. Recuperado el 3 de Abril de 2013, de Gallaudet Faculty-Staff Profile: http://www.gallaudet.edu/documents/academic/drl-dudis2007.pdf

DURANTI, A. (2000). Antropología Lingüística. Madrid: Cambridge University Press.

ECO, U. (2008). Decir casi lo mismo. Barcelona : Lumen.

ECO, U. (1996). Seis Paseos Por Los Bosques Narrativos. Madrid: Lumen.

EMMOREY, K. (2002). Language, Cognition, and the Brain: Insights From Sign Language Research. Mahwah, NJ: Lawrence Erlbaum Associates.

EMMOREY, K. (2003). Perspectives on Classifier Constructions in Sign Languages. Mahwah, NJ: Lawrence Erlbaum Associates.

FAUCONNIER, G. (1994). Mental spaces: Aspects of meaning construction in natural languages. Cambridge: Cambridge University Press.

GALVAN, B. D. (1988). The acquisition of three morphological subsystems in American Sign Language by deaf children with deaf or hearing parents. Tesis doctoral inédita, University of California, Berkeley.

GILE, D. (2009). Basic concepts and models for interpreter and translator training. Amsterdam: John Benjamins.

GONZÁLEZ MARTINEZ, H. (Ed.). (2014). La minificción en el siglo XXI: aproximaciones teóricas. Bogotá: Universidad Nacional de Colombia.

GUTT, E.-A. (2000). Translation and relevance. Oxford, UK: Blackwell.

HARRIS, B. (1980). Bi-text: A New Concept in Translation Theory. Language Monthly (54), 8-10. 
HARRIS, B. (1980). How a Three-Year-Old Translates. En E. A. Afendras (Ed.), Patterns of Bilingualism (págs. 370-393). Singapur: RELC.

HAUSER, P. C., FINCH, K. L., \& HAUSER, A. B. (Edits.). (2008). Deaf Professionals and Designated Interpreters: A New Paradigm. Washington: Galaudet University Press.

HERNÁNDEZ, L. F. (2002). Circo de Tres pistas y otros mundos mínimos. Ciudad de México: Ficticia.

HOLZ-MÄNTTÄRI, J. (1986). Translatorisches Handeln - theoretisch fundierte Berufsprofile. En M. Snell-Hornby, Übersetzungswissenschaft - Eine Neuorientierung. Zur Intergrierung von Theorie und Praxis (págs. 348 - 374). Tübingen: Francke.

HOLZ-MÄNTTÄRI, J. (1984). Traslatorisches Handeln. Theorie und Methode. Helsinki: Suomalainen Tiedeakatemia.

HYMES, D. (1974). Foundations in Sociolinguistics: An Ethnographic Approach. Philadelphia: University of Pennsylvania Press.

JACOBS, R. (1996). Just how hard is it to learn ASL: The case for ASL as a truly foreign language. En M. a. communities, \& C. Lucas (Ed.). Washington, DC: Gallaudet University Press.

JARRÍN BALLESTEROS, H. (2014). Cinco características de la posmodernidad en el relato breve. En H. Gonzalez Martinez (Ed.), La minificción en el siglo XXI: Aproximaciones teoricas (pág. 365). Bogotá: Universidad Nacional de Colombia.

LANGACKER, R. (2008). Cognitive Grammar: A Basic Introduction. New York: Oxford University Press.

LANGDON, C. (2011). Emergence of Depiction in Acquisition of American Sign Language. Poster Tesis doctoral, Gallaudet University, Linguistic, Lafayette, Indiana.

LIDDELL, S. (2003). Grammar, gesture and meaning in American Sign Language. Cambridge: Cambridge University Press. 
MCNEIL, D. (2005). Gesture and Thought. Chicago: Chicago University Press.

MALINOVSKY, B. (1935). Coral Gardens and Their Magic The Language and Magic of Gardening. New York: Routledge.

METZGER, M. (1995). Construed Dialogue and Construed Action in American Sign Language. En C. Lucas (Ed.), Sociolingüístics in Deaf Communities (Vol. 1, págs. 255 - 272). Washintong: Gallaudet University Press.

MONTERROSO, A. (1959). El Dinosaurio. En A. Monterroso, Obras Completas (y otros cuentos) (pág. 75). México DF: Joaquín Mortiz.

NORD, C. (1998). La unidad de traducción en el enfoque funcionalista. Quaderns: Revista de traducció , 65-77.

OBLER, L. K., \& GJERLOW, K. (2000). El lenguaje y el Cerebro. Madrid: Cambridge University Press.

OVIEDO, A. (2004). Classifier in Venezuelan Sign Language. Hamburg: Signum.

OVIEDO, A. (2001). Apuntes para una gramática de la lengua de señas colombiana. Bogotá: INSOR - UNIVALLE.

PACHECO, J. E. (1990). La sangre de Medusa. Ciudad de México: Era.

PADDEN, C. (1986). Verbs and Role-shifting in ASL. Proceedings of the 4th National Symposium on Signing Research and Teaching. Las Vegas, Nevada. Washington, DC: The National Association of the Deaf.

PENG, F. C. (1974). Kinship Signs in Japanese Sign Language. Sign Language Studies , 5, 31 - 47.

QUINTO-POZO, D. (2005). Factors that Influence the Acquisition of ASL for. En M. Marschark, R. Peterson, \& E. A. Winston (Edits.). Oxford: Oxford University Press. 
QUINTO-POZOS, D. (2007). Why Does Constructed Action Seem Obligatory? An Analisys of "Classifer" and the Lack of Articulator-Referent Correspondence. Sign Language Studies , 7 (4), 458 - 506.

REISS, K., \& VERMEER, H. (1996). Fundamentos para una teoría funcional de la traducción. Madrid: Akal.

SCHELLING, F. W. (1807 [1954]). Sobre la relación de las artes figurativas del arte con la naturaleza. Madrid: Aguilar.

SCHEMBRI, A. (2003). Rethinking 'Classifiers' in Signed Languages. En K. Emmorey (Ed.), Perspectives on Classifier Constructions in Sign Languages (págs. 3 - 34). Mahwah, New Jersey: Lawrence Erlbaum Associates.

SCHICK, B. (1990). The Effects of Morphosyntactic Structure on the Acquisition of Classifier Predicates. En C. Lucas (Ed.). Washington: Gallaudet University Press.

SLOBIN, D., HOITING, N., KUNTZE, M., LINDERT, R., WEINBERG, A., J., P., Y OTROS. (2003). A cognitive/functional perspective on the acquisition of "classifiers.". En K. Emmorey (Ed.), Perspectives on classifier constructions in sign languages (págs. 271 - 296). Mahwah, NJ:: Lawrence Erlbaum Associates.

SOUZA, S. X. (2010). A Norma Surda de Tradução em Ambientes Virtuais de Ensino e Aprendizagem: O Caso do Curso de Letras-Libras da UFSC. Recuperado el 23 de 04 de 2013, de II congresso nacional de pesquias em tradução e interpretação de LIBRAS y lingua portuguesa: http://www.congressotils.com.br/ anais2010/Saulo\%20Xavier\%20de\%20Souza.pdf

SPEBER, D., \& WILSON, D. (1986 (1994)). La relevancia. (E. Leonetti, Trad.) Madrid, España: Visor.

STONE, C. (2009). Toward a Deaf Translation Norm. Washintong: Gallaudet University Press.

THUMANN, M. (2011). Identifying depiction: Constructed Action and Constructed Dialogue. En Discourse in Signed Languaes (págs. 46-66). Washington: Gallaudet University Press. 
THUMANN, M. (2013). Identifying Recurring Depiction in ASL Presentations. Sign Language Studies , 13 (2), 316 - 349.

TOBÓN, S. (2005). Formación basada en competencias: Pensamiento complejo, diseño curricular y didáctica. Bogotá: ECOE Ediciones.

TOURY, G. (2004). Estudios descriptivos de la traducción y más allá. (R. Rabadán, Trad.) Madrid, España: Cátedra.

URBINA JOIRO, H. (2006). Entre las huellas de la India Catalina. Cartagena de Indias: Academia de la Historia de Cartagena de Indias.

WINSTON, E. (1996). Spatial Mapping in ASL Discourse. Assesing our work; 11th National Convention. Little Rock, Arkansas: CIT Proceedings.

WITTE, H. (2008). Traducción y Percepción Intercultural. Albolote (Granada): Comares.

ZABALA, L. (2005). La minificción bajo el microscopio. Bogotá: Universidad Pedagógica Nacional.

Recebido em: 02/07/2015

Aceito em: 22/09/2015 